\title{
Integrating Media Selection and Media Effects Using Decision Theory
}

\author{
Jacob T. Fisher ${ }^{1^{*}}$ \& Kristy A. Hamilton ${ }^{2}$
}

\begin{abstract}
Media psychology researchers seek to understand both why people choose certain media over others and how media influence cognitive, emotional, social, and psychological processes. A burgeoning body of literature has emerged in recent years describing media selection and media effects as reciprocally-linked dynamic processes, but research approaches empirically investigating them as such have been sparse. In parallel, technological developments like algorithmic personalization and mobile computing have served to blur the lines between media selection and media effects, highlighting novel problems at their intersection. Herein, we propose an integrative approach for building an understanding of these processes rooted in decision theory, a formal framework describing how organisms (and non-biological agents) select and optimize behaviors in response to their environment.
\end{abstract}

Keywords: computational modeling, theory development, media selection, media effects

Media psychology scholars have long labored to investigate both media selection (when and why people choose certain media behaviors over others), and media effects (the influence that media have on personal, social, and societal variables of interest). Although theorists acknowledge that media selection and media effects processes are mutually dependent (see, e.g. Lang \& Ewoldsen, 2009), extant research has, for the most part, considered the two processes in isolation from each other. Indeed, a recent meta-analysis of over 15,000 articles from media psychology and communication scholarship suggests that questions of selection and effects have served as central but largely distinct lines of inquiry over the last eight decades (Günther \& Domahidi, 2017). This is perhaps not surprising. The reciprocal relationship between the media-related choices that people make and the influence that media have in their lives is dynamic and idiosyncratic, with effects that span across multiple levels of analysis (Lang \& Ewoldsen, 2009; Sherry, 2015). In short, it is complex.

Although complexity is far from a new problem in media research (see, e.g. Berlo, 1960; Fink, 1993; Schramm, 1955), research approaches that consider "complexity as a matter of course" (Weber, Mathiak, et al., 2009, p. 50) still have not gained widespread traction in the field, thus limiting theory development at the intersection of selection and effects. Recent work has called for the adoption of formal modeling for developing theories of complex processes in a host of adjacent domains, including psychology, economics, and biology (Grahek et al., 2021; Guest \& Martin, 2021; van Rooij \& Baggio, 2021). In this manuscript, we provide an overview of formal modeling through the lens of decision theory (Dayan \& Daw, 2008; Körding, 2007), a formal framework designed to elucidate how organisms (and other decision-making entities) select and optimize their behaviors. Along the way, we outline important methodological and conceptual considerations involved in formalizing theories of media-related behaviors. Finally, we provide a brief example of the formalization process, constructing a toy model of task switching in a media multitasking environment that incorporates questions of both media selection and media effects.

\section{Navigating Complexity}

Media psychology research is not alone in its increasing acknowledgment of the complex nature of its objects of study. Recent years have seen mounting calls across the social and behavioral sciences for the development of new research paradigms that are more amenable to the study of complex psychological and social processes and that generalize to more naturalistic contexts (Muthukrishna \& Henrich, 2019; Sanbonmatsu \& Johnston, 2019). Several complementary strategies have been proposed to facilitate

1. Institute of Communications Research, College of Media, University of Illinois Urbana-Champaign

2. Department of Communication, University of California Santa Barbara

* Please address correspondence to jtfisher[at]illinois[dot]edu 
addressing this challenge. The first is the adoption of more "open" research and scholarship practices, including sharing data, pre-registering hypotheses, and conducting replication studies (Bowman \& Keene, 2018; Dienlin et al., 2021). The second is an increase in the use of "data-driven" tools and methods, coupled with greater emphasis on descriptive and inductive research (Han \& Lang, 2019; Van Atteveldt et al., 2019). The third is the expansion of more formal approaches to theory testing and development, pushing beyond purely verbal instantiations of social scientific theories to describe human behavior in terms of mathematical equations and/or algorithms (Grahek et al., 2021; Smaldino, 2020; van Rooij \& Baggio, 2021).

The first and second approaches outlined above have enjoyed a rapid uptick in adoption within the field, and their advantages have been well-discussed elsewhere (Bowman \& Keene, 2018; Dienlin et al., 2021). The final approach, although becoming more commonplace in cognate fields such as psychology, sociology, and human-computer interaction (Grahek et al., 2021; Guest \& Martin, 2021; van Rooij \& Baggio, 2021), has yet to take widespread root within communication and media psychology (although it has been periodically recommended, see e.g., Fink, 1993; Sherry, 2015). In this section, we provide a brief introduction to the process of formal modeling, followed by a discussion of the usefulness of formal models for explicating and testing theory. We focus on three main advantages of formal compared to traditional (verbal) approaches to theory development: 1 ) the inflexibility of formal models, 2) the fact that formal models generate point estimates for theoretical predictions, and 3) the ability of formal models to create bridges across different levels of analysis.

\section{A Formal Introduction}

By and large, theory development and testing in media psychology consists of two primary steps. First, the constructs germane to a theory and their relationships with one another are verbally articulatedoften with the help of graphical tools like boxes and arrows (Shoemaker et al., 2004). Next, support is generated for these theories by demonstrating predicted patterns of covariance between variables in a static snapshot of the processes of interest, such as an experiment or a survey (Poole, 2007). Generally, theoretically-predicted relationships are tested using null hypothesis significance testing (NHST; see, e.g. Levine, Weber, Hullett, et al., 2008; Levine, Weber, Park, et al., 2008), or related approaches, and a prediction is deemed "supported" if it can be shown that the likelihood of observing the predicted relationship under the null hypothesis is below a given threshold (often 5\%, but see Benjamin et al., 2018; Lakens et al., 2018).

In contrast, in a formal modeling approach, verbal descriptions of constructs and their relationships with one another are subjected to a process of formalization (Smaldino, 2020; van Rooij \& Baggio, 2021). The formalization process results in a mathematical (or computational) articulation of the variables of interest and their relationships with one another. Mathematical models are generally derived from a set of closed-form propositions and are supported with traditional proofs. Computational models, on the other hand, are formal descriptions of a process that may evolve over time or over another dimension (e.g., choices, events, trials) and can be iterated using computer code to generate simulated outputs (Farrell \& Lewandowsky, 2010; Smaldino, 2020; van Rooij \& Blokpoel, 2020). These simulated data can then be used to investigate the behavior of the system, how it might change under different parameterizations, and how well the model describes "real-world" data (Ballard et al., 2019; Farrell \& Lewandowsky, 2010).

Generally, formal models are described in terms of 1) a state - the composition of the system at a given point in time and/or the position of the agent within the environment, 2) a transition function - how the system moves through different states over time, and 3) a state-space - the set of all possible states to which the system could transition and the features of those states (Siegenfeld \& Bar-Yam, 2020; Strogatz, 2014). Different varieties of models can incorporate more specific terms, depending on the needs of the modeler. For example, computational models often describe agents that observe and explore the state space, actions that these agents might take (e.g., interacting with one another, learning features of the environment), and the goal(s) of the agent(s) at a given point in time (Jackson et al., 2017; Sutton \& Barto, 2018).

Once formalized, a model can be tested in several different ways. The first is via simulation and graphical inspection of model outputs (Vandekerckhove et al., 2015). Using simulations, researchers seek to judge whether the assumptions of the model are sufficient to produce the "behavior" of interest and that model outputs are generated as predicted based on the provided input values (Ballard et al., 2019). 
Next, researchers could design an experiment to manipulate parameters of interest (or locate a suitable publicly available dataset) and compare the predictions of the model to the data. Once quantitative data are generated, different models can be compared to one another (using Bayesian methods and/or crossvalidation; Rouder et al., 2018; Vehtari et al., 2017) to ascertain which model variant most accurately accounts for the data (without overfitting; see e.g., M. de Rooij \& Weeda, 2020; Yarkoni \& Westfall, 2017). At this step, researchers can add or remove individual parameters from the model, testing how different parameters influence the model's fit to the data, and facilitating consolidation (and competition) among alternative theories (Crockett, 2016; Jolly \& Chang, 2019). Finally, once a researcher has established that a model accurately accounts for the data, they can interpret the model's parameters to ascertain how changes in each parameter lead to changes in output variables of interest.

\section{Advantages of a Formal Approach}

Formal models, by their nature, are much less flexible than verbal models because all parts of the model must be specified in a way that can be computed algorithmically and/or calculated mathematically. This inflexibility is a strength in that it allows researchers to ensure that they are on the same page when communicating a model's assumptions and predictions (Smaldino, 2017). Although much can be accomplished through construct validation, clarification, and other efforts (DeAndrea \& Holbert, 2017; Grahek et al., 2021; Slater \& Gleason, 2012), the exact assumptions and predictions of verbally articulated models nonetheless run the risk of being misinterpreted or inconsistently applied. This is especially the case when different methods are used to test the predictions of the same model (Farrell \& Lewandowsky, 2010) when the same model is tested across different researchers or research groups (Guest \& Martin, 2021; van Rooij \& Baggio, 2021), or when the complexity of the system described by the model necessitates that analogies or "folk theories" be used to communicate about it (Jolly \& Chang, 2019; Rozenblit \& Keil, 2002).

Second, formal models enable researchers to generate point (or distribution) estimates that denote the expected value that each variable in the model will take and that vary as a function of the model's parameters. This stands in contrast to the hypothesis statements most commonly encountered in an NHST approach, which rather than directly positing a predicted distribution, posit that the distribution of an observed variable will be different from a null distribution (with a certain margin of error). Because of this, hypotheses derived from formal models are especially useful when researchers need to know what a model actually predicts, such as when examining the predictions of competing models or when estimating how a model's predictions may change under different assumptions (Jolly \& Chang, 2019; Meehl, 1967). In both scenarios, the precision afforded by formalization enables researchers to directly compare evidential support for different hypotheses using qualitative (e.g., plotting, visual inspection) and/or quantitative techniques (e.g. Bayesian model comparison, Vandekerckhove et al., 2015).

Finally, formal models allow researchers to create bridges across different levels of explanation. In recent work, Huskey and colleagues (2020) emphasize the utility of Marr's (1982) tri-level framework for generating an understanding of human media and communication behaviors. In this framework, explanation of human behavior is divided into three levels: computational, implementational, and algorithmic. Formal models exist at the algorithmic level, acting as "recipes" that translate inputs into outputs in view of the computational and implemental description of the system (Crockett, 2016; Niv \& Langdon, 2016). Because of this, formal models allow researchers to connect behavioral processes to the underlying neural and biological systems that enable the behavior of interest (Huskey et al., 2020; van Rooij \& Baggio, 2021). Perhaps of special interest to media psychology scholars, formal models also enable research approaches that bridge individual- and group-level processes, combining models of the behavior of individuals with agent-based models describing their interactions (Madsen et al., 2019; Waldherr \& Wettstein, 2019).

\section{Implementing a Formal Approach}

It is clear that an approach rooted in formal modeling affords much to the media psychology researcher interested in integrating questions of media selection and effects. This said, there are notable barriers in place when it comes to the widespread adoption of formal modeling in the field. The first is a lack of training in the tools required to generate and test formal models (Sherry, 2015). In addition to requiring mathematical expertise and coding skills, formal modeling introduces a host of new terminology and 
new questions that must be considered throughout the process (Farrell \& Lewandowsky, 2018). Although a number of excellent overviews of the formalization process have recently been introduced (see, e.g. Smaldino, 2020; Van Rooij \& Blokpoel, 2020), the sheer quantity of possible ways in which verbal theories can be formalized is an imposing obstacle to overcome-especially when considering the rich landscape of media selection and effects processes at play in a modern media environment.

One approach to reducing the difficulty inherent in adopting formal modeling is to start by "play[ing] around with" an existing model rather than developing a model from scratch (Smaldino, 2020, p. 208). This allows the interested researcher to get a sense of how each of the model's parameters contributes to its outputs and how the dynamics of the model progress under varying assumptions. Although there are undoubtedly numerous models that will prove to be useful within media psychology, those derived from decision theory (Dayan \& Daw, 2008; Körding, 2007) are perhaps an especially promising place to begin. This is for two primary reasons: First, decision theory affords a conceptual structure anchored in biological explanation (Cappella, 1996; Weber, Mathiak, et al., 2009) and spanning multiple levels of analysis (Huskey et al., 2020), thus affording researchers a common language when speaking across research subdomains and disciplines. Second, extant work in decision theory has provided a host of well-validated and welldocumented models (and software packages) that can considerably flatten the learning curve for the interested researcher. As such, decision-theoretic models can be employed in media psychology with relatively little overhead compared to that which would be required to develop (and code) formal models from scratch.

\section{Decision Theory}

Decision theory is a formal framework for describing, predicting, and explaining how organisms (and also non-biological agents) select certain behaviors in response to internal and external cues. In this framework, decisions are conceptualized as action-dependent transitions between states in view of a goal (Botvinick et al., 2015; Sutton \& Barto, 2018). Decision theory posits that agents seek to optimize their transitions between states by learning (and predicting) relative differences in reward or punishment proffered by currently available options (Dayan \& Balleine, 2002; Körding, 2007; Tavoni et al., 2019). This framework has been successfully employed to understand and predict behavior in humans, but also in a menagerie of other organisms, ranging from primates to prairie voles (Adams et al., 2012). Furthermore, decision theory is a central component in the burgeoning domains of machine learning and artificial intelligence research (Silver et al., 2017, 2018; Sutton \& Barto, 2018). As such, decision theory gives media psychology researchers a common language to discuss related problems across a number of different fields.

This formalization provides a powerful means of reducing the (quasi-infinite) "state space" of possible decisions into a small number of manipulable parameters (Crockett, 2016; Jolly \& Chang, 2019). But how can decision theory inform media selection and effects? Media use involves an almost endless assortment of decisions playing out across multiple timescales and often involving multiple concurrent goals (Sherry, 2015). A large body of media psychology research has sought to isolate and catalog the intrinsic (Tamborini et al., 2010; Weber, Tamborini, et al., 2009) or extrinsic (Reinecke et al., 2014) motivations underlying media use, but it is unclear how to consider individual motivations in this diverse patchwork in relation to one another. For example, how could a researcher develop a model when someone might choose to scroll social media versus play a video game when the motivations for doing either might be quite different? To answer this question, we must turn to the implementational level of decision theory.

Recent work from biology and the cognitive neurosciences provides a scaffold upon which to build understanding regarding how a large collection of heterogeneous motivations can be integrated into a single, unified process (Pearson et al., 2014; Schultz et al., 1997). This work has revealed that choice behaviors are mediated via a common neural "currency" (Grabenhorst \& Rolls, 2011; Levy \& Glimcher, 2012) or perhaps a directly encoded action policy (Hayden \& Niv, 2020) that allows a diverse set of potentially rewarding or threatening options to be compared along a single continuum. This "currency"-encoded primarily in the form of signals in the orbitofrontal and ventromedial prefrontal cortices, the ventral striatum, and the amygdalae-has been shown to mediate choices related to information foraging (Behrens et al., 2007; Kobayashi \& Hsu, 2019), cognitive effort investiture (Westbrook \& Braver, 2015, 2016), moral judgment 
(Crockett et al., 2017; Siegel et al., 2018), social learning (Burke et al., 2010), attention (Krauzlis et al., 2014), and monetary value (Gu et al., 2019), among many other things.

To review, decision theory answers the "why" (computational level) question by proposing that decisionmaking processes serve to select behaviors that are optimal given current goals and constraints (Tavoni et al., 2019). To answer the "how" (implementational level) question, decision theory proposes that decisionmaking processes are mediated by neural signals that encode information regarding the benefits and the costs of available options in a manner that enables comparison across diverse choice sets-a proposal with more than a decade of increasing empirical support (Basten et al., 2010; Levy \& Glimcher, 2012). In decision-theoretic research, these two levels are bridged by models regarding what rules might govern the decision-making process.

There are a large number of formal models (and variants thereof) that have been employed in decisiontheoretic research, but they can be divided into two general clusters: those focused on the process by which we learn and represent the relative value of available options, and those focused on the process by which we translate choice values into actual decisions. The first category of models can be contextualized within media research to theorize about the effects of media, conceptualizing these effects in terms of an associative learning process by which the relative value of different media behaviors is reinforced over time as a function of each behavior's association with positively or negatively weighted outcomes (Fisher et al., 2018). In turn, the second group of models can be used to develop theories regarding how these learned associations guide current or future media selections, modifying the likelihood that a particular option will be selected, the speed with which the decision is made, and other parameters that may be of interest in the decision-making process.

\section{Decision-Theoretic Models}

\section{Learning Reward Values}

One of the simplest and most influential formalizations of the reward learning (often called reinforcement learning) process is the Q-learning model (Watkins \& Dayan, 1992), which proposes that the predicted reward that an agent ascribes to a possible future state $Q_{i}(t+1)$ is updated based on discrepancies between the reward that is observed upon accessing a particular state $R_{i}(t)$ and the reward that was expected based on the previous time that the state was accessed $Q_{i}(t)$. This discrepancy is called the reward prediction error $(R P E)$. Thus:

$$
Q_{i}(t+1)=Q_{i}(t)+\left[\alpha_{L} \times\left(R_{i}(t)-Q_{i}(t)\right)\right]
$$

Here, $\alpha_{L}$ refers to the learning rate or gain (ranging from 0 to 1), describing how quickly the agent will adapt its representations to new information. When the learning rate is high, the represented value of a potential state changes rapidly in response to new information, whereas when it is lower, the value of a potential state is modulated slowly over many trials.

The Q-learning model is an example of a model-free learning process, in which the value of each available option in the current is learned in a context-free fashion and represented as a scalar number (Dayan \& Niv, 2008). In most modern models, value is also subjected to discounting based on uncertainty (Rushworth \& Behrens, 2008), costs (e.g., time, effort, opportunity, (e.g., time, effort, opportunity; Basten et al., 2010). The value of unchosen options can also be updated based on a forgetting rate, which gradually decays the value of unchosen options (Katahira, 2015). Although less common in formal modeling of human behavior, other model-free reward learning processes may also be used. These include policy optimization, which directly generates "action policies" rather than computing the value of available options, and the actorcritic model (Mnih et al., 2016), which combines reward learning and policy generation methods.

Reward values can also be learned in a model-based fashion, in which the agent seeks to optimize reward over a longer series of decisions or over a longer period of time (Dolan \& Dayan, 2013; Sutton \& Barto, 2018). To do this, the agent must learn both the rules of the task and the structure of the environment-creating an internal model that can be used to predict the value of other possible states (Gläscher et al., 2010). 
In practice, this internal model is typically represented as a matrix of transition probabilities between different states. T(s, a, s'), combined with the reward values of each state. Model-based reward learning is more computationally intensive than model-free learning but has been shown to be more effective in guiding goal-directed behavior (Dolan \& Dayan, 2013). Growing evidence suggests that human beings use both model-based and model-free methods to optimize decision-making strategies (Daw et al., 2011; S. W. Lee et al., 2014).

\section{Making Decisions}

To model how the agent makes a decision, the (learned) value parameter is incorporated into a decision function. One widely used decision function is the softmax (Sutton \& Barto, 2018), in which the relative value of each available option is converted into an action probability denoting the likelihood that a particular option will be chosen over another:

$$
P_{i}(t+1)=\frac{e^{Q_{i}(t)} \times \beta}{\sum_{k=1}^{N} e^{Q_{k}(t)} \times \beta}
$$

In this equation, the action probably for a given option(i)is a function of the reward value of that option $Q_{i}(t)$ divided by the sum of the reward values of all other available options. This function is modulated by a precision parameter, $(\beta)$, that determines the sensitivity of the choice likelihood to differences in reward values. This parameter can account for the fact that organisms vary in the extent to which they choose optimal behaviors, and that they sometimes make mistakes (Crockett, 2016; Friston et al., 2014). The precision parameter has been shown to be modulated by affective states (such as arousal; T.-H. Lee et al., 2012; Mather \& Sutherland, 2011), by cognitive differences (like ADHD; Hauser et al., 2016), and by characteristics of the environment (Behrens et al., 2007; Nassar et al., 2010).

One limitation of the softmax is that it is a static decision function, whereas many decision processes evolve continuously over time. As such, researchers may select another popular decision model that describes the dynamics of the decision-making process: the drift-diffusion model (DDM, Ratcliff \& McKoon, 2008; Ratcliff \& Smith, 2004). In the DDM, it is proposed that choices between tasks are the result of an evidence-accumulation process, in which a person gradually acquires evidence in favor of a particular choice compared to another (thus drifting toward that choice over time).

A DDM model contains a handful of parameters, most notably a starting point $(z)$, a drift rate $(v)$, boundary separation $(a)$, and non-decision time $\left(T_{e r}\right)$. The starting point of a DDM indicates the decision maker's bias toward one option over another. The drift rate indicates the speed at which the decision-maker acquires evidence for one decision for another. As such, the drift rate can be interpreted as the signal-to-noise ratio of the evidence accumulation process. A high drift rate indicates that the decision was of low difficulty (i.e., one option was very clearly more valuable than the other; Wagenmakers et al., 2007). Boundary separation, also referred to as a decision threshold, is an indicator of the conservativeness of the decision-maker when choosing a response-more conservative decision-makers have higher thresholds. Finally, non-decision time accounts for the time that is required for processes that are not related to the decision itself (e.g., perceptual or motor processes).

The drift-diffusion model is able to account for both choice likelihood and reaction time information across a wide range of decision paradigms in both humans and animals (for a review, see Ratcliff et al., 2016). In addition, parameters of the DDM have been shown to robustly relate to neural responses in both cognitive and social tasks (see e.g., Lin et al., 2020; Roberts \& Hutcherson, 2019). Multi-attribute versions of the DDM can account for evidence accumulation processes along multiple different continua (Busemeyer et al., 2019), and that account for the value of a particular choice for the self or for another person (Roberts $\&$ Hutcherson, 2019). In addition, the DDM can directly be combined with reward learning processes using the RLDDM (Pedersen et al., 2017), enabling researchers to capture the dynamics of both reward learning and decision making. 


\section{A Brief Example: Media Multitasking}

Decision theory provides a valuable theoretical framework and set of computational models for understanding human behavior. In this section, we provide a brief overview of what a decision-theoretic approach may look for a problem of interest in media psychology research. We focus on task switching, a component of media multitasking wherein individuals switch from one media task to another (Ophir et al., 2009; Wang et al., 2015). Task switching is an example of a process wherein media selection and media effects are intricately and dynamically intertwined. Even considering only a sparse set of variables, the likelihood that someone will select a particular media task depends on the state and trait characteristics of an individual, but also the person's expectations about the task (e.g. whether it be enjoyable, entertaining, etc). These expectations are formed in response to the effects that the mediated task has induced in the past (among other variables). Because the dynamics of the process are essential for understanding its function, task switching is an ideal candidate for developing a formal decision-theoretic model.

Initial work has already been done to conceptualize media switching behaviors in terms of motivated transitions between states (Wang et al., 2006, 2011). The ChaCha model, developed by Wang and colleagues (2006), proposes that the likelihood that a person will flip from one channel to another when watching television can be modeled as a logistic function that takes into account the "attractiveness" of a TV channel, the amount of time a person has been watching a particular channel, and a noise parameter. One could further formalize this model to state that a decision to switch from one mediated task (referred to as a state) to another that is within the current state space (see Figure 1a) will be dependent on: (a) the value that is associated with the current task, $Q_{i}(t),(2)$ the predicted value of an alternative task $Q_{j}(t)$, and, (3) the predicted cognitive resource cost (effort) required by both the current $E_{i}(t)$ and alternative task $E_{i}(t)$. This effort component can be modeled in terms of a discounting function (Kable \& Glimcher, 2007) that diminishes the value of a given state at a given rate $(\gamma)$ in relation to anticipated effort. This results in the following model for the value function:

$$
Q_{i}(t+1)=Q_{i}(t)+\left[\alpha_{L} \times\left(\left[R_{i}(t) \times \gamma^{E_{i}(t)}\right]-Q_{i}(t)\right)\right]
$$

To complete this toy model, a final parameter could be added to the decision function to account for individual differences in multitasking preferences. Recent work suggests that individual differences in self-reported media multitasking habits may predict variation in the precision parameter $(\beta)$ that is used during task switching behaviors, with high multitaskers engaging in more exploratory behaviors and low multitaskers engaging in more exploitative ones (Gong \& Duff, 2020). As such, one could modify the precision parameter of a softmax decision function to account for these individual differences (see Figure 1c). If $Q_{j}(t)$ is judged to be more valuable than $Q_{i}(t)$, one could expect that a person would be more likely to transition states (i.e., choose to engage in the alternative media) than to remain in the same state, especially if the precision parameter is high.

This model can be tested in a number of ways, some of which look quite similar to those used to test verbally articulated predictions and some of which are quite different. First, the outputs of the model could be graphically visualized to ascertain whether its parameters are sufficient to produce expected outputs (see Figure 1d). At this stage, model parameters could be tweaked to explore how outputs vary as a function of differing inputs. For example, one could change values for or to explore how different precision parameters or discounting rates influence the likelihood to switch tasks. A researcher could also design an experiment wherein the reward and effort values are manipulated for various media tasks and then fit the model (and model variants of interest) to the data that are collected. At this stage, rather than (or in addition to) generating support for the model with inferential statistical tests, support can be generated by directly comparing the accuracy of the model's predictions to those of competing models. This can be done using a number of approaches, including Bayesian model comparison or cross-validated accuracy metrics (such as precision and recall).

The model we present here, although rudimentary, provides an example of how a theory of switching between mediated tasks may be formalized and how decision theory can be used to build models that bridge processes related to media selection (via a decision function) and media effects (via reward learning 
and updating processes). As with any model, it is contingent upon a number of assumptions, including that people have the capacity to learn and represent the value of a quasi-infinite array of media behaviors, that media decisions are made in a context- and history-free fashion and that effort is the only relevant discounting factor when determining whether to switch media tasks. Of course, these assumptions are "wrong" in the strictest sense, but they are wrong in a way that can be tested and amended much more readily than is possible for the (often unspoken) array of assumptions that underpin verbal models (Smaldino, 2017). This means that, should another model be shown to be more accurate, or should an assumption of the proposed model be discarded (both of which are nearly certain to occur), a new model can be positioned in its place, allowing for a modular, iterative approach to developing theory in this domain.

A
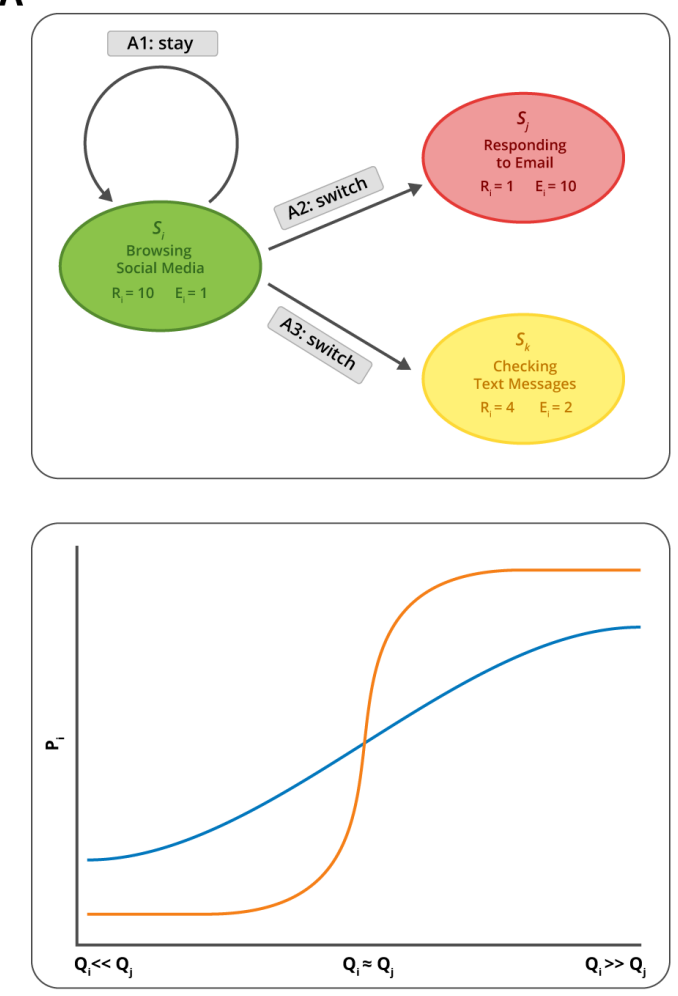

C

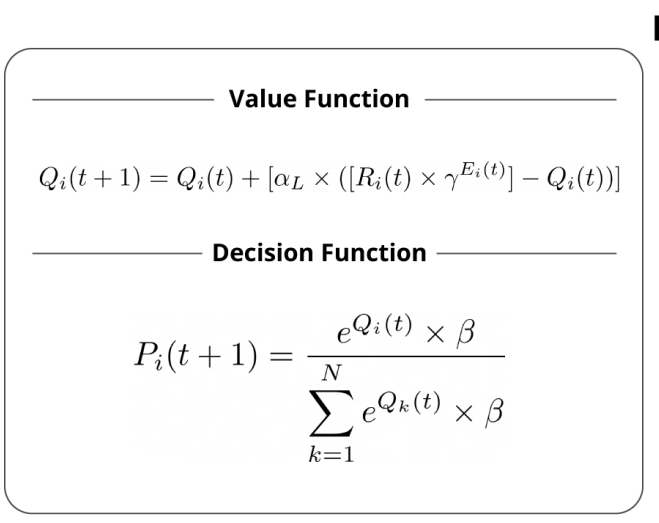

B

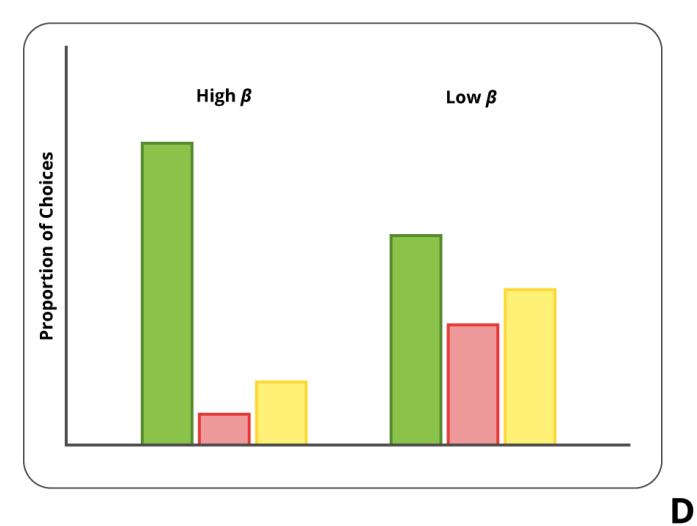

Figure 1: Depiction of a candidate decision-theoretic model for task-switching within the context of media multitasking behavior. (a) State diagram of a particular task-switching decision. States are shown as circles, and actions as gray rectangles. Arrows correspond to state transitions, and hypothetical reward outcomes are color-coded within their respective states. (b) Candidate value function for task switching behavior in a mediated context. The value of a given state updated as an integration of the predicted value of the state, the learning rate, and the effort-discounted reward that was obtained upon selecting the state. This value is combined with the values of all other available states in a decision function, resulting in the probability that the given state will be chosen from among the alternatives. (c) Graphical depiction of extreme values in the precision parameter $(\beta)$, which modulates the steepness of the softmax decision function. The $\mathrm{X}$ axis denotes the difference in values between states $Q_{i}$ and $Q_{j}$, and the $Y$ axis denotes the probability that the individual will select state $i$. Low beta values result in more exploratory behavior, and higher values result in more exploitative behavior. (d) Simulated outcomes resulting from the decision function for high and low precision parameter values, depicting proportional selections among the states at each level of $\beta$.

\section{Concluding Remarks}

Media psychology scholars have long described media selection and media effects as interconnected dynamic processes, but empirical approaches that consider them as such have been few and far between. Herein, we highlighted the usefulness of formal modeling for developing and testing theory at the intersection of selection and effects. We introduced decision theory as an integrative biologically-rooted framework that affords explanatory power across multiple levels of analysis and that provides a collection of well-validated computational models of both choice valuation (as a function of a learning process) and 
selection. To demonstrate the utility of this approach, we provided a candidate decision-theoretic model for media-related task-switching behavior based on the relative rewardingness and effortfulness of two competing tasks, combined with a person's multitasking preferences.

A key strength of formal modeling for understanding cognitive and social processes is that formalizing a theory requires a researcher to precisely articulate each assumption and prediction incumbent on the theory (Smaldino, 2020; van Rooij \& Baggio, 2021). Formal models must be explicit about each part of the system that is included (or excluded), as well as about how each of the parts functions and interacts. This, of course, means that formal models must "do some violence to reality" (Lazer \& Friedman, 2007), temporarily ignoring certain components of a system or certain edge cases in which the system behaves in a nonstandard way (Smaldino, 2017). In doing so, formal models sacrifice the comfortable shroud of strategic ambiguity that can buffer verbally articulated models against disconfirming evidence and alternative explanations (Muthukrishna \& Henrich, 2019). As such, formal models may seem clumsy or sparse compared to verbal models, but their precision and inflexibility allow them to serve as cognitive prosthetics aiding our ability to both reason about complex systems and to communicate about them (Farrell \& Lewandowsky, 2010).

Although formal modeling has clear benefits for cutting through complexity to develop more integrative and rigorous theory, a considerable amount of overhead is involved in adopting this approach. There are a practically infinite number of ways that media selection and media effects processes could be formally modeled, each of them requiring substantial mathematical and computer programming skills. As a potential inroad to developing more formal theories within media psychology, we introduced a collection of models derived from decision theory (Dayan \& Daw, 2008; Körding, 2007). These models are widely used across a formidable body of literature from biology to artificial intelligence, giving media psychology scholars a lingua franca facilitating bridge-building efforts across subfields within the discipline, but also between media psychology and other research domains. (Adams et al., 2012; Frankenhuis et al., 2019; Silver et al., 2018). Although still in its incipient stages, emerging work demonstrates the effectiveness of a decision-theoretic approach to understanding media-related processes, using computational models to predict social media engagement (Lindström et al., 2021), and to elucidate how online social learning processes may amplify the diffusion of moral outrage in online environments (Brady et al., 2021)

The need for media psychology scholars to develop formal models of complex media processes has perhaps never been more pressing. Recent technological developments like algorithmic personalization (Just \& Latzer, 2017; Napoli, 2014), and the rise of mobile, wearable, and "ambient" communication technologies (Vorderer et al., 2018), have drastically altered both how we select digital media content and the role that digital media play in our day-to-day lives. In an algorithmically personalized media environment, selection behaviors (e.g., reading an article, clicking a link, watching a video) can influence future selections via cognitive and social processes as described in extant models, but also via algorithmic processes that curate the selections that may be presented in the future (Bodó et al., 2019; Cappella et al., 2015). Furthermore, the increasingly personal, hyper-connected nature of modern digital tools facilitates deeply transactive relationships between humans and digital media that are not well-explained by traditional media selection and effects theories (Hamilton \& Benjamin, 2019; Hamilton \& Yao, 2018). To understand modern-and future-media behaviors, media psychology scholars must push forward in developing theories that can account for the complex interplay between cognitive, social, and algorithmic processes that characterize the modern media landscape. 


\section{References}

Adams, G. K., Watson, K. K., Pearson, J., \& Platt, M. L. (2012). Neuroethology of decision-making. Current Opinion in Neurobiology, 22(6), 982-989. https://doi.org/10.1016/j.conb.2012.07.009

Basten, U., Biele, G., Heekeren, H. R., \& Fiebach, C. J. (2010). How the brain integrates costs and benefits during decision making. Proceedings of the National Academy of Sciences, 107(50), 21767-21772. https://doi.org/10.1073/pnas.0908104107

Behrens, T. E. J., Woolrich, M. W., Walton, M. E., \& Rushworth, M. F. S. (2007). Learning the value of information in an uncertain world. Nature Neuroscience, 10(9), 1214-1221. https://doi. org/10.1038/nn1954

Benjamin, D. J., Berger, J. O., Johannesson, M., Nosek, B. A., Wagenmakers, E.-J., Berk, R., Bollen, K. A., Brembs, B., Brown, L., Camerer, C., Cesarini, D., Chambers, C. D., Clyde, M., Cook, T. D., De Boeck, P., Dienes, Z., Dreber, A., Easwaran, K., Efferson, C., ... Johnson, V. E. (2018). Redefine statistical significance. Nature Human Behaviour, 2(1), 6-10. https://doi.org/10.1038/s41562-017-0189-z

Berlo, D. (1960). Process of communication: An introduction to theory and practice. Harcourt School.

Bodó, B., Helberger, N., Eskens, S., \& Möller, J. (2019). Interested in diversity: The role of user attitudes, algorithmic feedback loops, and policy in news personalization. Digital Journalism, 7(2), 206229. https://doi.org/10.1080/21670811.2018.1521292

Botvinick, M., Weinstein, A., Solway, A., \& Barto, A. (2015). Reinforcement learning, efficient coding, and the statistics of natural tasks. Current Opinion in Behavioral Sciences, 5, 71-77. https://doi. org/10.1016/j.cobeha.2015.08.009

Bowman, N. D., \& Keene, J. R. (2018). A layered framework for considering open science practices. Communication Research Reports, 35(4), 363-372. https://doi.org/10.1080/08824096.2018.1513273

Brady, W. J., McLoughlin, K., Doan, T. N., \& Crockett, M. (2021). How social learning amplifies moral outrage expression in online social networks. PsyArXiv. https:// doi.org/10.31234/osf.io/gf7t5

Burke, C. J., Tobler, P. N., Baddeley, M., \& Schultz, W. (2010). Neural mechanisms of observational learning. Proceedings of the National Academy of Sciences, 107(32), 14431-14436. https://doi. org/10.1073/pnas.1003111107
Busemeyer, J. R., Gluth, S., Rieskamp, J., \& Turner, B. M. (2019). Cognitive and neural bases of multiattribute, multi-alternative, value-based decisions. Trends in Cognitive Sciences, 23(3), 251-263. https:// doi.org/10.1016/j.tics.2018.12.003

Cappella, J. N. (1996). Why biological explanation? Journal of Communication, 46(3), 4-7. https://doi. org/10.1111/j.1460-2466.1996.tb01485.x

Cappella, J. N., Kim, H. S., \& Albarracín, D. (2015). Selection and transmission processes for information in the emerging media environment: Psychological motives and message characteristics. Media Psychology, 18(3), 396-424. https://doi.org/10. 1080/15213269.2014.941112

Crockett, M. J. (2016). How formal models can illuminate mechanisms of moral judgment and decision making. Current Directions in Psychological Science, 25(2), 85-90. https://doi. org/10.1177/0963721415624012

Crockett, M. J., Siegel, J. Z., Kurth-Nelson, Z., Dayan, P., \& Dolan, R. J. (2017). Moral transgressions corrupt neural representations of value. Nature Neuroscience, 20(6), 879-885. https://doi.org/10.1038/ nn. 4557

Daw, N. D., Gershman, S. J., Seymour, B., Dayan, P., \& Dolan, R. J. (2011). Model-based influences on humans' choices and striatal prediction errors. Neuron, 69(6), 1204-1215. https://doi.org/10.1016/j. neuron.2011.02.027

Dayan, P., \& Balleine, B. W. (2002). Reward, motivation, and reinforcement learning. Neuron, 36(2), 285-298. https://doi.org/10.1016/S08966273(02)00963-7

Dayan, P., \& Daw, N. D. (2008). Decision theory, reinforcement learning, and the brain. Cognitive, Affective, \& Behavioral Neuroscience, 8(4), 429-453. https://doi.org/10.3758/CABN.8.4.429

Dayan, P., \& Niv, Y. (2008). Reinforcement learning: The good, the bad and the ugly. Current Opinion in Neurobiology, 18(2), 185-196. https://doi. org/10.1016/j.conb.2008.08.003

DeAndrea, D. C., \& Holbert, R. L. (2017). Increasing clarity where it is needed most: Articulating and evaluating theoretical contributions. Annals of the International Communication Association, 41(2), 168180. https://doi.org/10.1080/23808985.2017.1304163 
Dienlin, T., Johannes, N., Bowman, N. D., Masur, P. K., Engesser, S., Kümpel, A. S., Lukito, J., Bier, L. M., Zhang, R., Johnson, B. K., Huskey, R., Schneider, F. M., Breuer, J., Parry, D. A., Vermeulen, I., Fisher, J. T., Banks, J., Weber, R., Ellis, D. A., ... de Vreese, C. (2021). An agenda for open science in communication. Journal of Communication, 71(1), 1-26. https://doi.org/10.1093/joc/jqz052

Dolan, R. J., \& Dayan, P. (2013). Goals and habits in the brain. Neuron, 80(2), 312-325. https://doi. org/10.1016/j.neuron.2013.09.007

Farrell, S., \& Lewandowsky, S. (2010). Computational models as aids to better reasoning in psychology. Current Directions in Psychological Science, 19(5), 329-335. https://doi.org/10.1177/0963721410386677

Farrell, S., \& Lewandowsky, S. (2018). Computational modeling of cognition and behavior. Cambridge University Press.

Frankenhuis, W. E., Panchanathan, K., \& Barto, A. G. (2019). Enriching behavioral ecology with reinforcement learning methods. Behavioural Processes, 161, 94-100. https://doi.org/10.1016/j. beproc.2018.01.008

Friston, K., Schwartenbeck, P., FitzGerald, T., Moutoussis, M., Behrens, T., \& Dolan, R. J. (2014). The anatomy of choice: Dopamine and decisionmaking. Philosophical Transactions of the Royal Society B: Biological Sciences, 369(1655), 20130481. https://doi.org/10.1098/rstb.2013.0481

Gläscher, J., Daw, N., Dayan, P., \& O’Doherty, J. P. (2010). States versus rewards: Dissociable neural prediction error signals underlying modelbased and model-free reinforcement learning. Neuron, 66(4), 585-595. https://doi.org/10.1016/j. neuron.2010.04.016

Gong, X. J., \& Duff, B. R. L. (2020, May). An exploration account of media multitasking: The exploration-exploitation model to explain media multitasking behavior. Paper Presented at the 69th Annual Conference of the International Communication Association.

Grabenhorst, F., \& Rolls, E. T. (2011). Value, pleasure and choice in the ventral prefrontal cortex. Trends in Cognitive Sciences, 15(2), 56-67. https://doi. org/10.1016/j.tics.2010.12.004
Grahek, I., Schaller, M., \& Tackett, J. L. (2021). Anatomy of a psychological theory: Integrating construct-validation and computational-modeling methods to advance theorizing. Perspectives on Psychological Science, 1745691620966794. https://doi. org/10.1177/1745691620966794

Gu, R., Huang, W., Camilleri, J., Xu, P., Wei, P., Eickhoff, S. B., \& Feng, C. (2019). Love is analogous to money in human brain: Coordinate-based and functional connectivity meta-analyses of social and monetary reward anticipation. Neuroscience \& Biobehavioral Reviews, 100, 108-128. https://doi. org/10.1016/j.neubiorev.2019.02.017

Guest, O., \& Martin, A. E. (2021). How computational modeling can force theory building in psychological science. Perspectives on Psychological Science, 1745691620970585. https://doi. org/10.1177/1745691620970585

Günther, E., \& Domahidi, E. (2017). What communication scholars write about: An analysis of 80 years of research in high-impact journals. International Journal of Communication, 11, 30513071.

Hamilton, K. A., \& Benjamin, A. S. (2019). The human-machine extended organism: New roles and responsibilities of human cognition in a digital ecology. Journal of Applied Research in Memory and Cognition, 8(1), 40-45. https://doi.org/10.1016/j. jarmac.2019.01.001

Hamilton, K. A., \& Yao, M. Z. (2018). Cognitive offloading and the extended digital self. In $M$. Kurosu (Ed.), Human-Computer Interaction. Theories, Methods, and Human Issues (pp. 257-268). Springer International Publishing. https://doi. org/10.1007/978-3-319-91238-7_22

Han, J., \& Lang, A. (2019). It's a journey: From media effects to dynamic systems. Media Psychology, O(0), 1-21. https://doi.org/10.1080/15213269.2019.160423 6

Hauser, T. U., Fiore, V. G., Moutoussis, M., \& Dolan, R. J. (2016). Computational psychiatry of ADHD: Neural gain impairments across Marrian levels of analysis. Trends in Neurosciences, 39(2), 63-73. https://doi.org/10.1016/j.tins.2015.12.009

Hayden, B., \& Niv, Y. (2020). The case against economic values in the brain. PsyArXiv. https://doi. org/10.31234/osf.io/7hgup 
Huskey, R., Bue, A. C., Eden, A., Grall, C., Meshi, D., Prena, K., Schmälzle, R., Scholz, C., Turner, B. O., \& Wilcox, S. (2020). Marr's tri-level framework integrates biological explanation across communication subfields. JournalofCommunication, 70(3), 356-378. https://doi.org/10.1093/joc/jqaa007

Jackson, J. C., Rand, D., Lewis, K., Norton, M. I., \& Gray, K. (2017). Agent-based modeling: A guide for social psychologists. Social Psychological and Personality Science, 8(4), 387-395. https://doi. org/10.1177/1948550617691100

Jolly, E., \& Chang, L. J. (2019). The flatland fallacy: Moving beyond low-dimensional thinking. Topics in Cognitive Science, 11(2), 433-454. https://doi. org/10.1111/tops.12404

Just, N., \& Latzer, M. (2017). Governance by algorithms: Reality construction by algorithmic selection on the Internet. Media, Culture \& Society, 39(2), 238-258. https://doi. org/10.1177/0163443716643157

Kable, J. W., \& Glimcher, P. W. (2007). The neural correlates of subjective value during intertemporal choice. Nature Neuroscience, 10(12), 1625-1633. https://doi.org/10.1038/nn2007

Kaelbling, L. P., Littman, M. L., \& Cassandra, A. R. (1998). Planning and acting in partially observable stochastic domains. Artificial Intelligence, 101(1-2), 99-134. https://doi.org/10.1016/S00043702(98)00023-X

Katahira, K. (2015). The relation between reinforcement learning parameters and the influence of reinforcement history on choice behavior. Journal of Mathematical Psychology, 66, 59-69. https://doi.org/10.1016/j.jmp.2015.03.006

Kobayashi, K., \& Hsu, M. (2019). Common neural code for reward and information value. Proceedings of the National Academy of Sciences, 116, 13061-13066. https://doi.org/10.1073/pnas.1820145116

Körding, K. (2007). Decision theory: What "should" the nervous system do? Science, 318(5850), 606-610. https://doi.org/10.1126/science.1142998

Krauzlis, R. J., Bollimunta, A., Arcizet, F., \& Wang, L. (2014). Attention as an effect not a cause. Trends in Cognitive Sciences, 18(9), 457-464. https://doi. org/10.1016/j.tics.2014.05.008
Lakens, D., Adolfi, F. G., Albers, C. J., Anvari, F., Apps, M. A. J., Argamon, S. E., Baguley, T., Becker, R. B., Benning, S. D., Bradford, D. E., Buchanan, E. M., Caldwell, A. R., Van Calster, B., Carlsson, R., Chen, S.-C., Chung, B., Colling, L. J., Collins, G. S., Crook, Z., ... Zwaan, R. A. (2018). Justify your alpha. Nature Human Behaviour, 2(3), 168-171. https://doi. org/10.1038/s41562-018-0311-x

Lang, A., \& Ewoldsen, D. (2009). Beyond effects: Conceptualizing communication as dynamics, complex, nonlinear, and fundamental. In S. Allen (Ed.), Rethinking Communication: Keywords in Communication Research (pp. 109-120). Hampton Press.

Lazer, D., \& Friedman, A. (2007). The network structure of exploration and exploitation. Administrative Science Quarterly, 52(4), 667-694. https://doi.org/10.2189/asqu.52.4.667

Lazer, D., Pentland, A. (Sandy), Adamic, L., Aral, S., Barabasi, A. L., Brewer, D., Christakis, N., Contractor, N., Fowler, J., Gutmann, M., Jebara, T., King, G., Macy, M., Roy, D., \& Van Alstyne, M. (2009). Life in the network: The coming age of computational social science. Science (New York, N.Y.), 323(5915), 721-723. https://doi.org/10.1126/ science.1167742

Lee, S. W., Shimojo, S., \& O’Doherty, J. P. (2014). Neural computations underlying arbitration between model-based and model-free learning. Neuron, 81(3), 687-699. https://doi.org/10.1016/j. neuron.2013.11.028

Lee, T.-H., Itti, L., \& Mather, M. (2012). Evidence for arousal-biased competition in perceptual learning. Frontiers in Psychology, 3. https://doi.org/10.3389/ fpsyg.2012.00241

Levine, T. R., Weber, R., Hullett, C., Park, H. S., \& Lindsey, L. L. M. (2008). A critical assessment of null hypothesis significance testing in quantitative communication research. Human Communication Research, 34(2), 171-187. https://doi.org/10.1111/ j.1468-2958.2008.00317.x

Levine, T. R., Weber, R., Park, H. S., \& Hullett, C. R. (2008). A communication researchers' guide to null hypothesis significance testing and alternatives. Human Communication Research, 34(2), 188-209. https://doi.org/10.1111/j.1468-2958.2008.00318.x

Levy, D. J., \& Glimcher, P. W. (2012). The root of all value: A neural common currency for choice. Current Opinion in Neurobiology, 22(6), 1027-1038. https://doi.org/10.1016/j.conb.2012.06.001 
Lin, H., Saunders, B., Friese, M., Evans, N. J., \& Inzlicht, M. (2020). Strong effort manipulations reduce response caution: A preregistered reinvention of the ego-depletion paradigm. Psychological Science, 31(5), 531-547. https://doi. org/10.1177/0956797620904990

Lindström, B., Bellander, M., Schultner, D. T., Chang, A., Tobler, P. N., \& Amodio, D. M. (2021). A computational reward learning account of social media engagement. Nature Communications, 12(1), 1311. https://doi.org/10.1038/s41467-020-19607-x

Littman, M. L. (2009). A tutorial on partially observable Markov decision processes. Journal of Mathematical Psychology, 53(3), 119-125. https://doi. org/10.1016/j.jmp.2009.01.005

Madsen, J. K., Bailey, R., Carrella, E., \& Koralus, P. (2019). Analytic versus computational cognitive models: Agent-based modeling as a tool in cognitive sciences. Current Directions in Psychological Science, 28(3), 299-305. https://doi. org/10.1177/0963721419834547

Marr, D. (1982). Vision: A computational investigation into the human representation and processing of visual information. MIT Press.

Mather, M., \& Sutherland, M. R. (2011). Arousalbiased competition in perception and memory. Perspectives on Psychological Science, 6(2), 114-133. https://doi.org/10.1177/1745691611400234

Meehl, P. E. (1967). Theory testing in psychology and physics: A methodological paradox. Philosophy of Science, 34, 103-115.

Mnih, V., Badia, A. P., Mirza, M., Graves, A., Lillicrap, T. P., Harley, T., Silver, D., \& Kavukcuoglu, K. (2016, June 16). Asynchronous methods for deep reinforcement learning. Proceedings of the 33rd International Conference on Machine Learning. http:// arxiv.org/abs/1602.01783

Muthukrishna, M., \& Henrich, J. (2019). A problem in theory. Nature Human Behaviour. https://doi. org/10.1038/s41562-018-0522-1

Napoli, P. M. (2014). Automated media: An institutional theory perspective on algorithmic media production and consumption. Communication Theory, 24(3), 340-360. https://doi.org/10.1111/ comt.12039
Nassar, M. R., Wilson, R. C., Heasly, B., \& Gold, J. I. (2010). An Approximately Bayesian Delta-Rule Model Explains the Dynamics of Belief Updating in a Changing Environment. Journal of Neuroscience, 30(37), 12366-12378. https://doi.org/10.1523/ JNEUROSCI.0822-10.2010

Niv, Y., \& Langdon, A. (2016). Reinforcement learning with Marr. Current Opinion in Behavioral Sciences, 11, 67-73. https://doi.org/10.1016/j. cobeha.2016.04.005

Open Science Collaboration. (2015). Estimating the reproducibility of psychological science. Science, 349(6251). https://doi.org/10.1126/science.aac4716

Ophir, E., Nass, C., \& Wagner, A. D. (2009). Cognitive control in media multitaskers. Proceedings of the National Academy of Sciences, 106(37), 15583-15587. https://doi.org/10.1073/pnas.0903620106

Pearson, J. M., Watson, K. K., \& Platt, M. L. (2014). Decision making: The neuroethological turn. Neuron, 82(5), 950-965. https://doi.org/10.1016/j. neuron.2014.04.037

Pedersen, M. L., Frank, M. J., \& Biele, G. (2017). The drift diffusion model as the choice rule in reinforcement learning. Psychonomic Bulletin \& Review, 24(4), 1234-1251. https://doi.org/10.3758/ s13423-016-1199-y

Poole, M. S. (2007). Generalization in process theories of communication. Communication Methods and Measures, 1(3), 181-190. https://doi. org/10.1080/19312450701434979

Ratcliff, R., \& McKoon, G. (2008). The diffusion decision model: Theory and data for two-choice decision tasks. Neural Computation, 20(4), 873-922. https://doi.org/10.1162/neco.2008.12-06-420

Ratcliff, R., \& Smith, P. L. (2004). A comparison of sequential sampling models for two-choice reaction time. Psychological Review, 111(2), 333-367. https:// doi.org/10.1037/0033-295X.111.2.333

Ratcliff, R., Smith, P. L., Brown, S. D., \& McKoon, G. (2016). Diffusion decision model: Current issues and history. Trends in Cognitive Sciences, 20(4), 260281. https://doi.org/10.1016/j.tics.2016.01.007

Reinecke, L., Vorderer, P., \& Knop, K. (2014). Entertainment 2.0? The role of intrinsic and extrinsic need satisfaction for the enjoyment of facebook use. Journal of Communication, 64(3), 417438. https://doi.org/10.1111/jcom.12099 
Roberts, I. D., \& Hutcherson, C. A. (2019). Affect and decision making: Insights and predictions from computational models. Trends in Cognitive Sciences, 23(7), 602-614. https://doi.org/10.1016/j. tics.2019.04.005

Rouder, J. N., Haaf, J. M., \& Aust, F. (2018). From theories to models to predictions: A Bayesian model comparison approach. Communication Monographs, 85(1), 41-56. https://doi.org/10.1080/03637751.2017. 1394581

Rozenblit, L., \& Keil, F. (2002). The misunderstood limits of folk science: An illusion of explanatory depth. Cognitive Science, 26(5), 521-562. https://doi. org/10.1207/s15516709cog2605_1

Rushworth, M. F. S., \& Behrens, T. E. J. (2008). Choice, uncertainty and value in prefrontal and cingulate cortex. Nature Neuroscience, 11(4), 389397. https://doi.org/10.1038/nn2066

Sanbonmatsu, D. M., \& Johnston, W. A. (2019). Redefining science: The impact of complexity on theory development in social and behavioral research. Perspectives on Psychological Science, 14(4), 672-690. https://doi.org/10.1177/1745691619848688

Schramm, W. (1955). Information theory and mass communication. Journalism Quarterly, 32(2), 131146. https://doi.org/10.1177/107769905503200201

Schultz, W., Dayan, P., \& Montague, P. R. (1997). A neural substrate of prediction and reward. Science, 275(5306), 1593-1599. https://doi.org/10.1126/ science.275.5306.1593

Shannon, W. (1948). A mathematical theory of communication. Bell System Technical Journal, 27(3), 379-423.

Sherry, J. L. (2015). The complexity paradigm for studying human communication: A summary and integration of two fields. Review of Communication Research, 1, 22-65. https://doi.org/10.12840/ issn.2255-4165.2015.03.01.007

Shoemaker, P. J., Jr, J. W. T., \& Lasorsa, D. L. (2004). How to build social science theories. SAGE Publications.

Siegel, J. Z., Mathys, C., Rutledge, R. B., \& Crockett, M. J. (2018). Beliefs about bad people are volatile. Nature Human Behaviour, 2(10), 750. https://doi. org/10.1038/s41562-018-0425-1

Siegenfeld, A. F., \& Bar-Yam, Y. (2020, July 27). An introduction to complex systems science and its applications [Review Article]. Complexity; Hindawi. https://doi.org/10.1155/2020/6105872
Silver, D., Hubert, T., Schrittwieser, J., Antonoglou, I., Lai, M., Guez, A., Lanctot, M., Sifre, L., Kumaran, D., Graepel, T., Lillicrap, T., Simonyan, K., \& Hassabis, D. (2018). A general reinforcement learning algorithm that masters chess, shogi, and Go through self-play. Science, 362(6419), 1140-1144. https://doi.org/10.1126/science.aar6404

Silver, D., Schrittwieser, J., Simonyan, K., Antonoglou, I., Huang, A., Guez, A., Hubert, T., Baker, L., Lai, M., Bolton, A., Chen, Y., Lillicrap, T., Hui, F., Sifre, L., van den Driessche, G., Graepel, T., \& Hassabis, D. (2017). Mastering the game of Go without human knowledge. Nature, 550(7676), 354359. https://doi.org/10.1038/nature24270

Slater, M. D., \& Gleason, L. S. (2012). Contributing to theory and knowledge in quantitative communication science. Communication Methods and Measures, 6(4), 215-236. https://doi.org/10.1080 /19312458.2012.732626

Smaldino, P. E. (2017). Models are stupid, and we need more of them. In R. R. Vallacher, S. J. Read, \& A. Nowak (Eds.), Computational Social Psychology (1st ed., pp. 311-331). Routledge. https://doi. org/10.4324/9781315173726-14

Smaldino, P. E. (2020). How to translate a verbal theory into a formal model. Social Psychology, 51(4), 207-218. https://doi.org/10.1027/1864-9335/a000425

Strogatz, S. (2014). Nonlinear dynamics and chaos. Taylor \& Francis.

Sutton, R. S., \& Barto, A. G. (2018). Reinforcement learning: An introduction. MIT press.

Tamborini, R., Bowman, N. D., Eden, A., Grizzard, M., \& Organ, A. (2010). Defining media enjoyment as the satisfaction of intrinsic needs. Journal of Communication, 60(4), 758-777. https://doi. org/10.1111/j.1460-2466.2010.01513.x

Tavoni, G., Balasubramanian, V., \& Gold, J. I. (2019). What is optimal in optimal inference? Current Opinion in Behavioral Sciences, 29, 117-126. https:// doi.org/10.1016/j.cobeha.2019.07.008

Van Atteveldt, W., Margolin, D., Shen, C., Trilling, D., \& Weber, R. (2019). A roadmap for computational communication research. Computational Communication Research, 1(1), 1-11-1-11.

van Rooij, I., \& Baggio, G. (2021). Theory before the test: How to build high-verisimilitude explanatory theories in psychological science. Perspectives on Psychological Science, 1745691620970604. https://doi. org/10.1177/1745691620970604 
van Rooij, I., \& Blokpoel, M. (2020). Formalizing verbal theories. Social Psychology, 51(5), 285-298. https://doi.org/10.1027/1864-9335/a000428

Vandekerckhove, J., Matzke, D., \& Wagenmakers, E.-J. (2015). Model comparison and the principle of parsimony. In J. R. Busemeyer, Z. Wang, J. T. Townsend, \& A. Eidels (Eds.), Oxford handbook of computational and mathematical psychology (Vol. 1, pp. 300-317). Oxford University Press. https://doi. org/10.1093/oxfordhb/9780199957996.013.14

Vehtari, A., Gelman, A., \& Gabry, J. (2017). Practical Bayesian model evaluation using leave-one-out cross-validation and WAIC. Statistics and Computing, 27(5), 1413-1432. https://doi.org/10.1007/s11222016-9696-4

Vorderer, P., Hefner, D., Reinecke, L., \& Klimmt, C. (Eds.). (2018). Permanently Online, Permanently Connected: Living and Communicating in a POPC World. Routledge. https://www.routledge.com/ Permanently-Online-Permanently-ConnectedLiving-and-Communicating-in-a/Vorderer-HefnerReinecke-Klimmt/p/book/9781138245006

Wagenmakers, E.-J., Van Der Maas, H. L. J., \& Grasman, R. P. P. P. (2007). An EZ-diffusion model for response time and accuracy. Psychonomic Bulletin \& Review, 14(1), 3-22. https://doi.org/10.3758/ BF03194023

Waldherr, A., \& Wettstein, M. (2019). Bridging the gaps: Using agent-based modeling to reconcile data and theory in computational communication science. International Journal of Communication, 13, 3976-3999. https://doi.org/10.5167/uzh-186794

Wang, Z. J., Busemeyer, J. R., \& Lang, A. (2006). Grazing or staying tuned: A stochastic dynamic model of channel changing behavior. In R. Sun \& N. Miyake (Eds.), Proceedings of The 28th Annual Conference of the Cognitive Science Society \& the 5th International Conference of the Cognitive Science (pp. 870-875). Lawrence Erlbaum \& Associates.
Wang, Z. J., Irwin, M., Cooper, C., \& Srivastava, J. (2015). Multidimensions of media multitasking and adaptive media selection. Human Communication Research, 41(1), 102-127.

Wang, Z. J., Lang, A., \& Busemeyer, J. R. (2011). Motivational processing and choice behavior during television viewing: An integrative dynamic approach. Journal of Communication, 61(1), 71-93. https://doi.org/10.1111/j.1460-2466.2010.01527.x

Watkins, C. J. C. H., \& Dayan, P. (1992). Q-learning. Machine Learning, 8(3), 279-292. https://doi. org/10.1007/BF00992698

Watts, D. J. (2017). Should social science be more solution-oriented? Nature Human Behaviour, 1(1), 0015. https://doi.org/10.1038/s41562-016-0015

Weber, R., Mathiak, K., \& Sherry, J. L. (2009). The neurophysiological perspective in mass communication research. In M. Beatty, J. McCroskey, \& K. Floyd (Eds.), Biological dimensions of communication: Perspectives, methods, and research (pp. 43-73). Hampton Press.

Weber, R., Tamborini, R., Westcott-Baker, A., \& Kantor, B. (2009). Theorizing flow and media enjoyment as cognitive synchronization of attentional and reward networks. Communication Theory, 19(4), 397-422. https://doi.org/10.1111/ j.1468-2885.2009.01352.x

Westbrook, A., \& Braver, T. S. (2015). Cognitive effort: A neuroeconomic approach. Cognitive, Affective, \& Behavioral Neuroscience, 15(2), 395-415. https://doi.org/10.3758/s13415-015-0334-y

Westbrook, A., \& Braver, T. S. (2016). Dopamine does double duty in motivating cognitive effort. Neuron, 89(4), 695-710. https://doi.org/10.1016/j. neuron.2015.12.029 